\title{
Economia e ldentidade no Século XXI: uma Análise das Contribuições de Akerlof e Kraton, Sen e Davis
}

\section{Economics and ldentity in the 21st Century: an Analysis of Contributions by Akerlof and Kraton, Sen and Davis}

\author{
Maríndia Brites ${ }^{\mathrm{a}, \mathrm{b}}$ [D \\ Solange Regina Marin ${ }^{\mathrm{C}}$ i \\ Felipe Almeida ${ }^{\mathrm{a}}$ (i)
}

\begin{abstract}
Resumo: O objetivo deste artigo é apresentar as principais abordagens econômicas contemporâneas que tratam do conceito de identidade. Essas abordagens são: a) o modelo mainstream de Akerlof e Kranton, no qual a identidade é uma externalidade que limita ou maximiza a utilidade dos indivíduos por meio da interação social; b) a abordagem de Amartya Sen, que associa as identidades ao commitment, uma forma de comportamento humano em contraposição ao indivíduo maximizador do autointeresse; e c) a identidade social de John Davis, oriunda das identidades pessoais e individuais, de modo que o comportamento humano englobe aspectos pessoais, mas que mantenha a individualização do indivíduo inserido em contextos sociais. $\mathrm{O}$ texto também apresenta um debate sobre como as três abordagens destacam a questão da identidade nos estudos econômicos. Esse debate é focado no tema tomada de decisão e considera como elementos psicológicos e sociológicos foram inseridos nas abordagens.
\end{abstract}

Palavras-chave: Identidade social. Commitment. George Akerlof. Amartya Sen. John Davis.

\begin{abstract}
The goal of this paper is introducing key economics' approaches that deal with the concept of identity. The key approaches are: a) Akerlof and Kranton's mainstream model of which takes identity into account as associate it with an externality that limits or maximizes the utility of individuals through social interaction; b) Amartya Sen's approach that relates identities toe commitment, which is a form of human behavior opposed to maximizing individual self-interest; c) the social identity of John Davis, it comes from the personal and individual identities, so that human behavior encompasses personal aspects, but leaving the individualization of individuals inserted in social contexts. The paper also introduces a debate on how the three approaches emphasize that the concept of "identity" should be embedded in economic studies. This debate relies on decision-making and considers how psychological and sociological elements were inserted into the approaches.
\end{abstract}

Keywords: Social identity. Commitment. George Akerlof. Amartya Sen. John Davis.

\footnotetext{
a Universidade Federal do Paraná (UFPR), Programa de Pós-Graduação em Desenvolvimento Econômico (PPGDE). Curitiba, Paraná, Brasil.

b Faculdade de Educação Superior do Paraná (FESPPR), Graduação em Ciências Econômicas, Curitiba, Paraná, Brasil.

c Universidade Federal de Santa Catarina (UFSC), Programa de Pós-Graduação em Economia. Florianópolis, Santa Catarina, Brasil.
} 
JEL Classification: D01; B59; Z13.

\section{Introdução}

Como qualquer outra ciência, a Economia é uma ciência em constante evolução (no sentido Darwiniano, que difere de aprimoramento). Obviamente, o mesmo é verdade para os conceitos e abordagens econômicas. Um processo evolucionário implica trajetórias dependentes de conceitos e análises. Por exemplo, a Economia gerou a tradição de considerar o indivíduo como elemento analítico central, mas sem considerar o que é um indivíduo, onde está inserido e quem é (KIRMAN; TESCHL, 2004). Além disso, na Economia a racionalidade do indivíduo tornou-se sinônimo de lógica otimizadora, diferente de outras ciências sociais (SIMON, 2000). Alguns pensadores econômicos e/ou escolas do pensamento econômico buscaram alternativas à "racionalidade econômica", como é o caso de John Maynard Keynes e a escola pós-keynesiana, Thorstein Veblen e o institucionalismo americano e Karl Marx e a economia marxista.

Independente da alternativa à tradicional racionalidade econômica, existe um foco em comum: a necessidade de considerar mais características do tomador de decisão ou do ambiente no qual está inserido. Este estudo repousa sobre um debate sobre outra alternativa à racionalidade econômica, considerar o papel da identidade na tomada de decisão. O conceito de identidade é cientificamente amplo, envolve diferentes abordagens oriundas, primeiramente, da Psicologia e da Filosofia e, posteriormente, da Antropologia e Sociologia, sendo um conceito complexo e de difícil definição. De forma genérica, a identidade tem ligação com o reconhecimento do tomador de decisão sobre a sua similaridade com outros tomadores de decisão.

A evolução das abordagens econômicas no entorno da racionalidade econômica gera dificuldades para incorporar o conceito de identidade ao estudo da tomada de decisão do indivíduo em uma perspectiva econômica - embora no passado a identidade estivesse presente, mesmo que não identificada de tal forma, em escritos de expressivos pensadores econômicos, tais como Adam Smith e John Stuart Mill.

Para Sen (2007), a identificação com os outros, em diferentes formas, pode ser extremamente importante para viver em sociedade. Porém, não tem sido fácil persuadir os cientistas econômicos contemporâneos para acomodar a identidade de forma satisfatória. Em particular, existem dois diferentes tipos de reducionismos na literatura formal das análises econômicas atuais. Uma é a não consideração das identidades - boa parte da teoria econômica contemporânea procede como se as pessoas no processo de escolha de seus objetivos e prioridades não possuíssem nenhuma identificação com outras pessoas, a não ser com si mesmas. Outra é 
denominada de afiliação única - qualquer pessoa pertence apenas a uma única coletividade.

Este trabalho apresenta uma análise das três principais abordagens econômicas atuais sobre os indivíduos e a sociedade que antecipa questões importantes no que diz respeito ao conceito de identidade na economia. ${ }^{1}$ Essas abordagens são: a) o modelo mainstream de Akerlof e Kranton, que incorpora a identidade na função de utilidade e expande a análise do mainstream; b) a abordagem de Amartya sem, cujo modelo incorpora o conceito de commitment, que relaciona os indivíduos com os grupos sociais; e c) a identidade social de John Davis pautada na análise filosófica e ontológica, que aborda níveis sociais, individuais e pessoais da identidade. Cada perspectiva analítica é apresentada em um item, respectivamente. Após a apresentação dessas abordagens, é introduzido um debate a respeito. Por último, seguem as considerações finais.

\section{A Abordagem de Akerlof e Kranton}

Definir aquilo que pode ser considerado o mainstream de uma ciência não é tarefa fácil. Isso não é diferente para a ciência econômica. É comum notar a utilização do termo "economia neoclássica" como sinônimo ao mainstream. No entanto, contemporaneamente é possível perceber que pressupostos da economia neoclássica não fazem parte do atual mainstream, como, por exemplo, a existência de informação perfeita - que foi colocada em cheque com a publicação de Akerlof (1970). Com o avanço da economia comportamental em estudos como tomada de decisão, nota-se que até a hipótese do comportamento otimizador dos tomadores de decisão nem sempre se faz presente em estudos do mainstream. O discurso do economista David Colander como presidente da History of Economics Society ilustra muito bem a dissociação necessária entre os termos economia neoclássica e mainstream. Esse discurso presidencial foi proferido em 1999 e, posteriormente, publicado por Colander (2000). O artigo de Colander (2000) é intitulado "A morte da economia neoclássica" (em tradução livre) e o seu ponto central é o termo "economia neoclássica", um objeto de análise da história do pensamento econômico. O que é produzido atualmente na economia não é economia neoclássica.

Talvez a definição de mainstream de Dequech seja esclarecedora: o mainstream pode ser considerado o conjunto de ideias que possuem a maior influência e prestígio na profissão. Os artigos publicados em revistas de prestígio e o conteúdo ensinado nas mais famosas universidades são baseados no mainstream

$\overline{1}$ A introdução da identidade permite definir o indivíduo como um agente que muda e aprende, é autorreflexivo, avalia as suas ações e é capaz de influenciar o seu entorno. Assim, o indivíduo é um ser com uma história, mas que muda e afeta conscientemente a própria autopercepção e as suas características pessoais, enquanto um ser distinto dos demais (KIRMAN; TESCHEL, 2004). 
(DEQUECH 2007, 2012). Colocados esses pontos, é seguro afirmar que o atual mainstream pode ser considerado uma evolução da economia neoclássica, mas não pode ser confundido com esta. ${ }^{2}$ Dentro dessa concepção de mainstream, o conceito de identidade - elemento central do presente artigo - foi introduzido pelos estudos de George Akerlof e Rachel Kranton. ${ }^{3}$ De acordo com Akerlof e Kraton (2000, p. 715), a identidade é "a person's sense of self". Para Akerlof e Kranton (2005), a identidade captura como os indivíduos se sentem sobre si mesmos e como esses sentimentos dependem de suas ações: "[...] the word identity to describe both a person's self-image as well as her assigned categories [...]" (AKERLOF; KRANTON, 2000, p. 718).

De acordo com Akerlof e Kranton (2000 e 2002), a importância da identidade para a análise econômica repousa sobre a explicação de fenômenos que o atual mainstream (sem considerar a identidade), por sua concepção teórico-metodológica, não consegue abordar a contento, como, por exemplo, escolarização e gênero. Nas palavras de Akerlof e Kranton (2005, p. 38): "[w]e see identity as the next step in the evolution of the economic [...]" - por economia, os autores entendem o mainstream. Logo, a grande contribuição de Akerlof e Kranton é expandir o escopo analítico do mainstream através da identidade. Os autores muitas vezes recorrem à incorporação de elementos de outras ciências ao modelo de tomada de decisão do mainstream, como, por exemplo, o modelo de Akerlof e Kranton (2000), que é baseado na teoria da psicodinâmica da personalidade comum na psicologia, enfatiza a importância na interiorização das regras para o comportamento.

De acordo com Akerlof e Kranton (2000), a inclusão do conceito de identidade no mainstream expande a análise de quatro maneiras distintas:

a) percepção: a identidade explica comportamentos que podem parecer prejudiciais. Sem se considerar a identidade, alguns comportamentos seriam considerados mal adaptados ou autodestrutivos. Considerando-se a identidade, tais comportamentos por ser adjetivados como sugerido por indivíduos que possuem outras identidades. No entanto, considerando-se as identidades dos tomadores de decisão, tais comportamentos podem ser interpretados como perfeitamente adaptados e benéficos aos indivíduos;

2 Nesse contexto, evolução carrega o seu significado Darwinista, ou seja, não implica necessariamente em melhora, mas em uma causação dinâmica e cumulativa.

3 George Arthur Akerlof é professor da Universidade da Califórnia em Berkeley, foi laureado com o Prêmio Nobel de Economia em 2001 por seu trabalho citado no texto, que descreve os problemas dos mercados com assimetria de informação (AKERLOF, 1970). Vale destacar que Michael Spence e Joseph Stiglitz também contribuíram profundamente para a inserção de assimetria de informação em estudos econômicos (SPENCE, 1973; STIGLITZ; ROTHSCHILD, 1976). Rachel Kranton é professora da Duke University. O artigo em questão é intitulado "Economics and identity". 
b) externalidade: a identidade pode ser considerada um tipo de externalidade, de modo que as ações do indivíduo podem influenciar as ações de outros indivíduos. Por exemplo, Akerlof e Kranton (2000) citam a questão de gênero: um vestido é considerado sinônimo de feminilidade; se um homem vestir um vestido, pode ameaçar a identidade dos outros homens. Portanto, há uma externalidade, e outras externalidades podem surgir se esses homens reagirem;

c) mudanças nas preferências: a identidade mostra uma nova maneira de como as preferências podem mudar. Segundo Akerlof e Kranton (2000), as identidades evoluem dentro da sociedade, e a própria sociedade pode incentivar essa evolução. Por exemplo, uma propaganda pode ditar o padrão de mulher e homem ideal;

d) autodefinição: como a identidade é fundamental para o comportamento humano, escolher a identidade, ou seja, escolher quem o indivíduo deseja ser pode ser a decisão econômica mais importante. Akerlof e Kranton (2000) enfatizam que os limites impostos sobre a escolha das identidades dos indivíduos podem ser também os determinantes mais importantes do bem-estar econômico dos indivíduos.

Na visão de Akerlof e Kranton (2002, 2005), a incorporação da identidade é uma ruptura com a tradição do mainstream, pois demanda uma modificação na composição da função utilidade. Levando-se a identidade em consideração, as funções de utilidade são construídas tanto por preferências quanto por identidades. As segundas implicam que funções utilidades podem mudar de acordo com categorias sociais, por exemplo. Além disso, espaço e tempo também podem implicar modificações. Para os autores, algumas identidades, como sexo, raça e nacionalidades, são comuns na vida cotidiana e, inclusive, são consideradas pela economia mainstream em forma de variáveis dummy.

Os modelos de Akerlof e Kranton modificam o método através do qual tais elementos são analisados por causa do seu tratamento como identidade. Identidades vão muito além da incorporação de variáveis dummy, identidades podem/ devem ser incorporadas na função utilidade como uma motivação para o comportamento dos indivíduos (AKERLOF; KRANTON, 2000). Na função utilidade, a identidade é baseada em categorias sociais, por exemplo, "homem" e "mulher", que são caracterizadas pelas prescrições, que mostram qual comportamento o tomador de decisão considera apropriado para a sua identidade. Por exemplo, uma mulher pode ter uma identidade mais feminina e culminar na motivação da utilização de um vestido. Um homem pode carregar consigo uma identidade mais masculina e não ser motivado a utilizar um vestido. É importante notar que tal abordagem permite a liberdade de sexismo, pois uma mulher pode escolher uma identidade mais masculina e um homem uma identidade mais feminina. 
Para se ilustrar mais claramente o impacto da identidade na tomada de decisão tradicional do mainstream, outros exemplos são destacados. Um exemplo ilustrativo repousa sobre a importância da supervisão do trabalhador na execução de suas tarefas. Akerlof e Kranton $(2005,2008)$ afirmam que os trabalhadores sob forte supervisão tendem a empregar menos esforço e, até mesmo, sabotar a produção das firmas. Para os autores, isso ocorre porque o trabalhador pode se identificar como parte do grupo de trabalho, mas, quando os trabalhadores são monitorados, isso pode criar um conflito entre empresa e trabalhador, que adota uma identidade em oposição à empresa, um problema que não seria solucionado apenas com aumento salarial - resposta da economia tradicional do mainstream a tal problema (a seguir, seguem maiores comentários sobre essa questão do incentivo monetário). ${ }^{4}$

Akerlof e Kranton (2008) ressaltam que essa análise da identidade não se restringe a modelos e que pode ser encontrada na prática, como nos locais de traba-

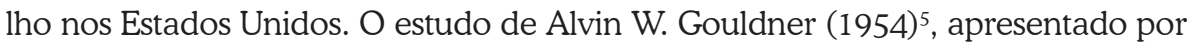
Akerlof e Kranton (2008), mostra que, após a substituição do supervisor de uma empresa, que mantinha o monitoramento ameno (os trabalhadores eram livres para passear na fábrica, recebiam regalias e havia pouca repressão em relação a atrasos: percebeu-se satisfação dos trabalhadores quanto ao local de trabalho), houve uma mudança de identidade por parte dos trabalhadores. O gerente substituto era ativo e os trabalhadores eram monitorados sob regime restrito de supervisão, o que despertou uma identidade contrária dos trabalhadores às políticas da empresa, gerando conflitos entre empresa e funcionários.

Akerlof e Kranton $(2005,2008)$ ressaltam que o conceito de identidade na abordagem econômica pode alterar a compreensão das políticas, comportamentos organizacionais e políticas de emprego, pois considerar apenas as motivações monetárias se torna ineficaz. Por exemplo, um trabalhador que se identifica com a empresa que trabalha requer menos pagamento: redução do salário médio e aumento de vantagem no investimento da identidade dos trabalhadores (AKERLOF; KRANTON, 2005, 2008). A identidade é a principal motivação dos indivíduos e

$4 \quad$ O paralelo entre a perspectiva de Akerlof e Kranton e o Nobel Herbert Simon pode ser ilustrativo. Para Simon (1991), a teoria neoclássica, ao considerar o lucro máximo como a única motivação dos indivíduos, leva a uma função de utilidade irrealista e que não retrata as motivações reais dos indivíduos em organizações. Segundo o autor, quatro fenômenos organizacionais centrais devem ser incluídos: autoridade, recompensa, coordenação e identificação. Em relação à identificação, assim como Akerlof e Kranton (2005, 2008), Simon (1991) ressalta que as pessoas são fortemente motivadas pela fidelidade à organização e o sentimento de orgulho em trabalhar na firma, ou seja, a identificação com a organização permite aos indivíduos obter satisfações, em forma de utilidade, dos sucessos da própria organização. Nas palavras de Simon (1991, p. 43): “[...] [t]he primacy of profit as the enforcer of organizational efficiency is replaced by organizational goals, combined with organizational identifications and with material rewards and supervision, all of which motivate employees to work toward these goals.".

5 GOULDNER, A. W. Patterns in industrial bureaucracy. Glencoe: The Free Press, 1954. 
pode contribuir para o bom funcionamento das empresas porque altera a compreensão das políticas empresariais, como o pagamento de incentivos e supervisão. Akerlof e Kranton (2005) entendem que o bom funcionamento das organizações não depende exclusivamente de políticas monetárias, mas também da capacidade das organizações de distribuir os trabalhadores em atividades com as quais se identificam.

Para Akerlof e Kranton $(2000,2005)$ a incorporação da identidade na função de utilidade amplia a análise do mainstream: com a identidade é possível analisar a motivação dos indivíduos de formas diferentes que vão além de questões monetárias. Por exemplo, Akerlof e Kranton (2002) propõem outra perspectiva para a análise da educação realizada pelo mainstream que ultrapassa os elementos investigados pelos modelos econômicos tradicionais, como recursos e incentivos, que não são suficientes para explicar as grandes diferenças em resultados educacionais (aprendizagem dos alunos) com pouca diferença na quantidade de recursos. O modelo econômico proposto por Akerlof e Kranton (2002) introduz elementos (identidade e categoria social), frequentemente omitidos pela economia do mainstream, logo podem explicar os resultados não captados por um modelo econômico convencional.

Nesse modelo, a função de utilidade do estudante contém, como principal motivação, a identidade. Com a identidade inserida no modelo, o estudante não visa apenas a maximização do valor presente do retorno monetário a seus estudos, mas busca também identificar-se com as categorias sociais que existem na escola (AKERLOF; KRANTON, 2002). A principal conclusão de Akerlof e Kranton (2002) é que políticas que modificam as categorias sociais e as prescrições podem ser mais eficazes nos resultados educacionais do que políticas que possuem como elementos principais os recursos e incentivos monetários para professores, dado que tais políticas não monetárias também alteram o comportamento dos indivíduos.

Adicionalmente, Akerlof (2002) afirma que a economia do mainstream também é incapaz de explicar a desvantagem dos negros americanos no que tange à pobreza e à criminalidade. Para o autor, esses indivíduos (negros) fazem escolhas sobre qual identidade desejam exercer: uma identidade que se adapte à cultura dominante (branca) ou a identidade alternativa, isto é, a identidade não dominante (negra). Se escolher a identidade dominante, o indivíduo tem conhecimento de que não será aceito plenamente pelos membros do grupo dominante, além de poder diminuir a autoimagem do indivíduo, que passa a ser alguém diferente do seu grupo de origem. Podem existir também ações da família e amigos que serão contra a alteração da identidade do indivíduo. Se optar pela segunda opção, a autoimagem do indivíduo dentro do seu grupo de origem (não dominante) é au- 
mentada, porém é degradada tanto em termos econômicos, quanto em físicos na cultura dominante.

Resumidamente, a inserção da identidade no modelo mainstream de Akerlof e Kranton (2000) contribui para a melhor compreensão de como o tomador de decisão cria uma autoimagem de si e como isso é incorporado na função de utilidade. Akerlof e Kranton (2002, 2005, 2008) têm por objetivo complementar a análise dos fenômenos econômicos, enriquecendo a economia do mainstream, pois o modelo capta tanto a escolha dos indivíduos com motivações monetárias quanto da sua identidade. Akerlof e Kranton (2000) não rompem o mainstream, buscam aperfeiçoá-lo. Porém, Akerlof e Kranton (2000) não foram os únicos que buscaram incorporar a identidade na análise econômica. O Nobel Amartya Sen (2005, 2009) também apresenta uma contribuição ao tema. Em contraste com Akerlof e Kranton (2000), Sen $(2005,2009)$ propõe uma modificação mais radical à abordagem do mainstream, por entender a maximização da utilidade como parte da teoria do comportamento humano, e não como a sua totalidade.

\section{A Abordagem do Commitment de Amartya Sen}

Amartya Sen aborda a identidade na economia com o termo commitment, que é considerado uma forma diferente de comportamento, que vai além do autointeresse (DAVIS, 2004, 2006) ${ }^{6}$. Para Sen (1989, 2002a), a teoria econômica tradicional está assentada sobre três pressuposições do "auto" ("self"), que são amplamente discutíveis: self-centered welfare (bem-estar autocentrado), self-welfare goal (bem-estar pessoal como objetivo) e self-goal choice (escolha focada em objetivos pessoais). O primeiro supõe que o bem-estar da pessoa depende apenas do seu próprio consumo e outras riquezas da sua vida; não envolve outros indivíduos; o segundo refere-se ao interesse do indivíduo de sempre maximizar o seu próprio bem-estar, isto é, a sua satisfação; e, por último, a escolha do indivíduo é inteiramente feita a fim de buscar seus próprios objetivos, sem levar em consideração outros indivíduos. Sen (1977) introduz um quarto conceito de "auto" que se associa com a ideia de os indivíduos serem capazes de formar comprometimentos com os outros indivíduos. $\mathrm{O}$ autor denominou esse conceito de commitment - que possui uma proximidade com aquilo que Sen denomina como simpatia e antipatia (SEN, 1977).

A simpatia/antipatia ocorre quando a preocupação com os outros afeta diretamente o próprio bem-estar do indivíduo. O commitment refere-se à escolha de

6 Amartya Kumar Sen é um economista indiano e ganhador do Prêmio Nobel de Economia, em 1998, por sua contribuição à economia do bem-estar. Sen (1989) chama a atenção para a negligência da identidade na economia moderna. Para o autor, isso ocorre pela restrição que o pressuposto de comportamento autointeressado impõe à Economia. 
uma pessoa agir em prol de outro, mesmo que essa ação implique um menor nível de bem-estar pessoal para o indivíduo - ou seja, exclui ações ligadas ao autointeresse. Além disso, o commitment está atrelado a questões morais, engloba influências religiosas e políticas (SEN, 1977, 2002b). Com a introdução do commitment, Sen (2002b) ressalta que os indivíduos são influenciados pelo contexto social em que estão inseridos e pelas pessoas que o indivíduo se identifica.

Hausman e McPherson (2006) e Sen (1977, 2008a), no entanto, não excluem o autointeresse como uma motivação das ações dos indivíduos, mas entendem que não é a única: os indivíduos também são guiados por questões morais, políticas, sociais e éticas. ${ }^{7}$ Sen (1998) liga o commitment com a identidade social ou com a afiliação social, que é como um indivíduo se identifica com outros de um determinado grupo. Para Sen (1998), a identidade social dos indivíduos é uma influência significativa para o comportamento humano; o indivíduo segue regras e comportamentos do grupo com o qual se identifica, sem relação com seus próprios objetivos. O comportamento baseado em commitment pode alterar a escolha do indivíduo à medida que reconhece os objetivos de outras pessoas, que são incorporados nos próprios objetivos do indivíduo (SEN, 2005). Sen (2005) ressalta que a introdução do commitment na teoria econômica é importante para o entendimento da racionalidade e também para explicar as variações no comportamento humano entre diferentes sociedades através do tempo; compreende melhor o comportamento dos indivíduos.

Entretanto, Sen (2009) ressalta que, além da identidade social, os indivíduos também possuem identidade pessoal, que não devem ser consideradas igualmente e nem devem ser agregadas em uma "identidade global/individual", como sugere Parekh (2009a, 2009b). ${ }^{8}$ Como "Personal identity is concerned with our being 'the same person' [...]” (SEN, 2009, p. 286), as prescrições da identidade pessoal são diferentes das características da identidade social que os indivíduos podem mudar inúmeras vezes de afiliações sociais sem se tornarem pessoas diferentes. Qizilbash (2014) argumenta que, na obra de Sen, é possível distinguir diferentes concepções de identidade: a autopercepção ou identidade pessoal, que é a forma como a pessoa vê a si mesma, as afiliações sociais ou identidade social, que são as identificações que o indivíduo tem como membro da família, partido político ou

7 Por exemplo, segundo Therét (2003), o institucionalismo histórico considera que os indivíduos tanto calculam com base em seus interesses como também possuem diferentes visões de mundo, que corresponde às suas crenças e contextos sociais.

8 Para Parekh (2009a, 2009b), a identidade pessoal engloba as crenças e o comprometimento do indivíduo em relação ao mundo em que vive. A identidade social, que é pluralista, aborda as relações das quais o indivíduo se identifica e se considera como um integrante. Ambas se relacionam e constituem a vida do indivíduo, que seria empobrecida caso houvesse separação entre identidade pessoal e social. Ambos formam o que Parekh (2009a, 2009b) nomeia de identidade global ou identidade individual, dado que, enquanto a identidade pessoal influencia a definição e priorização dos indivíduos sobre suas identidades sociais, a identidade social amplia o senso de si mesmo do indivíduo ao incluir outros indivíduos. 
outro grupo e a identidade percebida (perceived identity), ou seja, a forma como uma pessoa é percebida pelos demais indivíduos.

A identidade pessoal tem relação com a abordagem das capacitações que Sen (1985) propõe como alternativa à utilidade para avaliar o bem-estar, que trata de um critério objetivo e impessoal, tendo como partes constituintes os funcionamentos e as capacitações. Os funcionamentos consideram como a pessoa "funciona", ou seja, os "seres" e "fazeres", que podem ser atividades como comer, ler, ou estados de ser, como estar bem nutrido, estar livre de doenças. Um conjunto de funcionamentos que uma pessoa realiza é um vetor de funcionamentos e, conforme Sen (1985, p. 198), “[...] [t]he primary feature of a person's well-being is the functioning vector that he or she achieves [...]". O conjunto capacitário da pessoa é um conjunto de vetores de funcionamentos e está estritamente ligado com a liberdade que a pessoa possui de levar o tipo de vida que valora. Sob essa ótica, ao analisar o bem-estar dessa pessoa, verifica-se o conjunto de capacitações que o indivíduo possui para realizar os funcionamentos valorados (SEN, 1985). O autor ressalta que o bem-estar da pessoa pode ser analisado em termos de liberdade e de realização, ou seja, liberdade de bem-estar e liberdade de agência, e realização de bem-estar e realização de agência.

A liberdade de bem-estar se concentra na capacidade de a pessoa ter vários vetores de funcionamentos e ter realizações de bem-estar. A liberdade de agência é um conceito mais amplo de liberdade e relaciona-se com o aspecto de agência do indivíduo. A liberdade de agência considera que o indivíduo é livre para perseguir seus objetivos e valores, isto é, a liberdade de agência é a liberdade de a pessoa realizar o que considera valioso para si, sendo ou não relacionado com o seu bem-estar, como um agente responsável (SEN, 1985). Sen (1985) resgata o aspecto de agência e afirma que se trata também do próprio julgamento do indivíduo como um agente responsável, e não apenas como um seguidor do bem-estar.

A liberdade de agência é a chave para se compreender a identidade pessoal de Sen. A liberdade de agência considera a autonomia e a liberdade pessoal, que se relacionam com a capacitação de tomar decisões sobre a própria vida e ser capaz de escolher para si mesmo características básicas da sua existência pessoal (SEN, 1985). Para Sen (1998), é inaceitável negar a escolha quando esta existe, pois envolve a renuncia de considerar e avaliar a forma de pensamento e de identificação dos indivíduos. A escolha é importante e possível tanto na conduta individual quanto nas decisões sociais. Para Sen (1998), o indivíduo não possui apenas uma única identidade, possui múltiplas identidades que podem conflitar em determinadas situações.

Cada uma das identidades individuais pode ser importante para o indivíduo, depende da sua situação, do seu problema e da sua escolha. As prioridades das identidades são influenciadas pelos próprios valores e/ou pelas pressões sociais 
que os indivíduos sofrem (SEN, 2008b). Ainda segundo Sen (2008b), admitir que o indivíduo tenha uma única identidade é considerado um reducionismo de comportamento humano. Todos os indivíduos se veem como membros de uma variedade de grupos. Nas palavras de Sen (2002a, p.153): “[...] [t]he robbing of our plural identities not only reduces us, it impoverishes the world [...]".

Sen (2014) destaca a importância de se considerar a multiplicidade de identidades dos indivíduos, dado que estes pertencem a diferentes grupos e que as identidades são escolhidas pelos indivíduos, ao invés de ser imposta uma única identidade. Sen (1989) desloca a percepção do autointeresse como única motivação humana para levar em consideração as influências que as identidades sociais têm sobre os indivíduos, que amplia o conceito de indivíduo, antes considerado como independente de qualquer influência social.

Com relação à identidade pessoal, o objetivo de Sen é apresentar um indivíduo reflexivo capaz de avaliar a si mesmo como um agente e influenciar a sociedade em que vive. Com a introdução da sua abordagem de identidade na economia, Sen (2002b) consegue retratar melhor o bem-estar dos indivíduos no sentido de afirmar que as pessoas não estão apenas em busca do seu próprio bem-estar, mas também do bem-estar dos outros, assim como as pessoas não apenas estão preocupadas com os outros indivíduos, como o próprio bem-estar da pessoa depende dos outros (KIRMAN; TESCHL, 2004). Como Sen (2002b), Davis (2006) argumenta que, com a introdução da identidade, há o enriquecimento do conceito de indivíduo na economia à medida que se incorpora ao comportamento do indivíduo aspectos pessoais, individuais e sociais.

\section{A Identidade de John Davis}

John Davis possui como preocupação recorrente de pesquisa o indivíduo e seu comportamento no âmbito filosófico e ontológico. ${ }^{9} \mathrm{~A}$ abordagem utilizada por Davis (2003) para investigar o indivíduo na Economia é, denominada por ele, abordagem de critério-ontológico para identidade. Primeiramente, o autor pergunta o que o conceito de indivíduo requer ou quais critérios fundamentais estão envolvidos ao se referir a elementos do indivíduo. Após, verifica se certas concepções do indivíduo em Economia capturam esses requisitos e satisfazem esses critérios em relação a como foram formulados. Para Davis (2005), o indivíduo tem papel central na explicação do sistema econômico, porém ressalta que o conceito

9 John Bryan Davis é doutor em Filosofia e Economia e professor da Marquette University e da Universidade de Amsterdã. Foi editor da Review of Social Economy de 1987 a 2005, presidente da History os Economics Society e vice-presidente da European Society for the History of Economic Thought. Atualmente é editor do Journal of Economic Methodology. 
padrão de indivíduo não fundamenta uma teoria que explique o que significa ser um indivíduo.

Há razões para acreditar que o conceito de individualidade utilizado na Economia pode ser tido como inadequado: a concepção tradicional do indivíduo não explica o que é o indivíduo, apenas fornece uma concepção abstrata que utiliza o termo "individual" para representar os indivíduos como átomos, pessoas únicas, coleções de pessoas, países, máquinas ou qualquer conceito que seja possível atribuir uma função de maximização (DAVIS, 1992; 2003, 2011).

Davis (2009) afirma que a identidade não é um conceito familiar na economia, mas possui importância por pelo menos dois motivos. A maximização da utilidade tradicional no conceito de indivíduo na economia aceita que as preferências não mudam, são estáticas e não explica como os indivíduos mudam e fazem escolhas ao longo das suas vidas, supondo que os indivíduos são sempre os mesmos. Com a introdução da identidade, os indivíduos não são mais considerados seres estáticos, a mudança faz parte de suas vidas. A identidade também é importante para a aproximação entre a ética e a economia, por considerar o agente como um ser moral e responsável. A identidade torna-se importante para a compreensão do comportamento humano, levanta questionamentos a respeito da relevância da responsabilidade moral do indivíduo sobre suas escolhas econômicas.

Davis (2006) argumenta que a introdução da identidade na economia implicaria uma maior complexidade do conceito de indivíduo, no sentido de incorporar diferentes formas de comportamento que estão relacionadas também com a interação com os outros indivíduos. Para o autor, o comportamento humano considerado na Economia pode ser enriquecido se aceitos aspectos individuais e sociais, pois a individualidade é endógena à interação social. Kirman e Teschl (2004) afirmam que o interesse de Davis está em mostrar como um indivíduo incorporado socialmente pode construir a própria subjetividade, isto é, como o indivíduo constitui a identidade pessoal dentro de um ambiente social. ${ }^{10}$

Davis (2009) descreve as identidades como um complexo caracterizado por aspectos pessoais e individuais, que juntos formam as identidades sociais. $\mathrm{O}$ autor distingue a identidade pessoal da individual, afirma que muitos autores que tratam da identidade as consideram iguais ou simplesmente não as distinguem. A identidade pessoal refere-se a primeira pessoa do singular (eu), leva em consideração os aspectos particulares de cada indivíduo, de um ponto de vista único e com origem determinada, oriundas da reflexão pessoal do indivíduo, e é construída pelos próprios indivíduos (DAVIS, 2009). A identidade pessoal dos indivíduos, ao contrário

10 Kirman (2011) destaca que, dentre as ideias que embasam seu livro, uma delas é insatisfação com o homo economicus e com a inquietação sobre o que precisamente entendemos por identidade de um agente econômico. Para Kirman (2011), a estrutura de interação entre as pessoas é de suma importância para a economia. Destaca que a ideia de um mercado anônimo no qual as pessoas interagem somente por meio do sistema de preços parece ser totalmente não realística. 
das identidades individuais determinadas socialmente por outros, é determinada individualmente, embora sob a influência das concepções sociais de suas identidades individuais.

A identidade individual, por outro lado, refere-se a terceira pessoa do singular (ele), os indivíduos falam de muitas perspectivas ou de muitas concepções sociais de identidade dos indivíduos, surge de muitos locais e não de um ponto de vista único, como a identidade pessoal, e é oriunda das reflexões dos outros indivíduos (identificação com os outros). A identidade individual é construída socialmente, por diferentes membros de grupos, e a identidade pessoal faz parte da individual (DAVIS, 2009).

Para Davis (2009), há na identidade individual concepções sociais. Como exemplo de identidade individual, o autor cita os registros de identificação dos eleitores para evitar o voto duplo, os registros de saúde para aplicação de terapias de saúde consistentes, os registros de desempenho educacional para determinar a formação e o emprego do indivíduo, o sistema de pensão para equilibrar as contribuições e os rendimentos de aposentadoria, as notações de crédito para avaliação das finanças pessoais e as identificações biométricas para diversos usos. Nas palavras de Davis (2009, p. 89): “[...] [e]ach of these individual identity systems occupies distinct (although sometimes overlapping) social spaces, while their different rationales preclude their respective conceptions of individual identity from being reduced to one another [...]".

Para Davis (2009), os diferentes conceitos de identidade individual encontrados na sociedade são na realidade estados atribuídos aos indivíduos por outros indivíduos como objetos caracterizados de alguma forma (identificadores ou registros de identificação). Como as caracterizações dos indivíduos são produto de sistemas sociais que mudam lentamente, as identidades individuais tendem também a ter mudança lenta. Por outro lado, o conceito de identidade pessoal se refere a uma atividade que o indivíduo exerce como um agente. Essa atividade implica indivíduos com suas próprias caracterizações de suas identidades pessoais, que constroem a ideia de potencial para mudança ou para automudança.

Davis (2009) identifica quatro pontos de diferença entre os conceitos de identidade pessoal e individual: a) as concepções de identidade pessoal e individual podem entrar em conflito; b) as concepções de identidade individual são mais estáveis do que a identidade pessoal, que é mais passível de mudanças; c) as concepções de identidade individual tendem a incorporar algumas características das identidades pessoais; e d) embora as identidades individuais incorporem as identidades pessoais, estas não devem ser reduzidas à identidade individual, ou seja, identidades individuais e pessoais não são iguais.

A identidade pessoal (eu) e a identidade individual (ele) formam a identidade social (nós), entendida geralmente como sendo uma questão de indivíduos se 
identificarem com os outros membros do grupo (DAVIS, 2009). ${ }^{11}$ Porém, o conceito de identidade social possui dois significados distintos. O primeiro é compatível com a ideia de Aristóteles: o indivíduo possui um conjunto único de características associadas com "ser" alguma coisa. A identidade social é entendida como algo que o indivíduo possui como característica, como ser alto em estatura, ser residente em algum lugar, pertencer a um grupo relacionado com gênero, nacionalidade, religião ou ocupação (DAVIS, 2010).

O segundo conceito refere-se a algo que é resultado de alguma atividade, como o indivíduo se identificar com um grupo social. Se esse conceito for aceito, a identificação com os outros elimina a individualidade. $\mathrm{O}$ indivíduo deixa de ser distinto (separado) para ser parte de um grupo social. Davis (2010) afirma que, como alternativa a esse conceito restritivo, há uma visão mais moderada da ideia de identificação, que pode manter a individualidade, mas permite também que essa independência seja influenciada pela identificação social. O conceito de identidade social, utilizado por Davis (2010), se refere a essa visão moderada que permite ao indivíduo se identificar com os outros e ainda permanecer um indivíduo distinto dos demais.

Além disso, Davis (2010) enfatiza que a identidade social não deve apenas ser compreendida no sentido de os indivíduos se identificarem com os outros em grupos sociais, mas também que essa identificação ocorre simplesmente com os outros indivíduos, sem relação com grupos especificamente. A identidade social pode abranger diferentes níveis: com grandes grupos sociais, como se identificar com outras pessoas por sexo ou religião, com grupos sociais de dimensão intermediária, como no local de trabalho ou vizinhança, e com pequenos grupos sociais, como amigos ou família. Davis (2010), como Sen (1998) e March (1994), também argumenta contra considerar a identidade social do indivíduo como única, dado que o indivíduo se identifica simultaneamente com diferentes grupos sociais.

Davis (2009) explica que as identidades sociais dos indivíduos podem entrar em conflito com as suas identidades pessoais e individuais. Para o autor, a chave para entender esses conflitos pode ser encontrada na abordagem da psicologia social sobre a identidade social, conhecida como teoria da identidade social, como nos estudos de Henri Tajfel (1972) e John Turner (1985). Para esses autores, os indivíduos têm autoimagens que refletem as categorias sociais geradas no discurso social. Os indivíduos, em seguida, alteram seu comportamento em relação a ou-

11 Davis (2010) resgata três tentativas de abordar a identidade social, porém sem exatamente utilizar o termo "identidade": (i) o teorema da impossibilidade de Arrow; (ii) o teorema do observador imparcial de Harsanyi da ética social; e (iii) o indivíduo representativo de Lucas na análise macroeconômica. Por outro lado, para o autor, o modelo neoclássico de Akerlof e Kranton (2000), a abordagem do commitment de Sen (1977) e a análise de complexidade de Kirman e Teschl (2004) são abordagens contemporâneas que consideram as identidades sociais de diferentes formas. 
tros indivíduos conforme as características que os indivíduos do grupo social no qual o indivíduo se identifica possuem.

A abordagem de Tajfel (1972 apud DAVIS, 2009) ${ }^{12}$ e Turner (1985 apud DAVIS, 2009) ${ }^{13}$, para Davis (2009), constrói o conceito da identidade social como um elo entre a identidade pessoal e a individual, embora entenda que os autores não enfatizam a identidade pessoal. As identificações sociais dos indivíduos criam conflito entre suas identidades individuais como membros do grupo e também com suas próprias concepções de identidade pessoal. Como membros do grupo, os indivíduos têm um status de objeto criado pelos outros na manipulação da identidade pessoal. Porém, os indivíduos estão envolvidos em explicar a si mesmos quando suas ações os colocam em grupos sociais. Tanto as identificações sociais quanto as identidades individuais limitam a liberdade dos indivíduos de serem capazes de formular as imagens que possuem de si mesmos, ou seja, restringem as suas identidades pessoais.

Para retratar a importância das identidades sociais, Davis (2009) aborda o trabalho de Clark (1997) ${ }^{14}$ sobre andaimes socioinstitucionais. Clark (1997 apud DAVIS, 2009) discorre sobre a inteligência artificial e a teoria de redes neurais para compreender como os agentes humanos interagem nos ambientes. Para o autor, os cérebros humanos são muito bons em algumas atividades, como o reconhecimento de padrões, mas, por outro lado, não são muitos bons em outras atividades, como em cálculos matemáticos complexos. O argumento de Clark (1997 apud DAVIS, 2009) é que o cérebro humano é limitado em certas atividades, de forma que as funções cognitivas humanas mais fracas são transferidas para dispositivos externos (andaimes) a fim de aumentar a eficiência.

Os andaimes socioinstitucionais são estruturas externas que apoiam a cognição interna do indivíduo, ou seja, as instituições sociais criam andaimes para diferentes atividades do indivíduo que não podem ser realizadas muito bem pelo cérebro humano em ambientes institucionais. Davis (2009) argumenta que os andaimes socioinstitucionais não são puramente externos aos indivíduos, que, de certa forma, foram responsáveis por criá-los e influenciá-los, modificando e analisando a importância que os andaimes possuem para a facilitação das atividades humanas. A ênfase de Davis (2009) é que há uma relação interativa entre andaimes e indivíduos, ou seja, os andaimes socioinstitucionais modificam as atividades

12 TAJFEL, H. Social categorization. In: MOSCOVICI, S. (ed.). Introduction à la psychologie sociale. Paris: Larousse, 1972. v. 1, p. 272-302.

13 TURNER, J. Social categorization and the self-concept: a social cognitive theory of group behavior. In: LAWLER, E. (ed.). Advances in group processes: theory and research. Greenwich: JAI, 1985. v. 2, p. 77-122.

14 CLARK, A. Being there: putting brain, body and world together again. Cambridge: MIT Press, 1997. 
humanas e são influenciados pelos indivíduos. "This close interactive relationship thus effectively embodies human activities in social structures." (DAVIS, 2009, p. 91).

Para Davis (2009), os andaimes socioinstitucionais devem ser interpretados como identidades sociais que criam identidades individuais, além de operarem como dispositivos que modificam e formam as identidades pessoais. Como a identidade pessoal dos indivíduos reflete, no mínimo, o conteúdo de diferentes sociedades, as identidades individuais são parâmetros para a identidade pessoal.

Por fim, a ideia de Clark (1997 apud DAVIS, 2009) é que os indivíduos têm exteriorizado diferentes funções cognitivas, das quais o cérebro humano não é tão bom, com o intuito de melhorar as atividades. As diferentes concepções de identidade individual são exteriorizações de identidades pessoais que são mais bem desenvolvidas através de processos sociais. Como exemplo, Davis (2009) cita os registros de desempenho educacional, o boletim. O desenvolvimento pessoal é de suma importância para o indivíduo baseado na sua própria identidade pessoal, então os andaimes são construídos a partir da construção social das estruturas que são utilizadas para organizar e formular o desenvolvimento pessoal.

Em suma, para Davis (2003), o comportamento humano deve compreender os aspectos pessoais que englobam as autoimagens de cada indivíduo, mas também deve compreender a individualização das pessoas, que, no entanto, também interagem em sociedades e nos grupos sociais. A evolução na Economia, com a introdução de identidade na abordagem de Davis, ocorre através da complexidade do conceito do indivíduo, constituído por elementos pessoais, individuais e sociais. Nesse sentido, mais informações sobre indivíduo, para Davis (2011), contribui para a afirmação de que os indivíduos são seres sociais e que recebem influências do comportamento dos demais indivíduos, alterando a percepção que têm de si mesmos.

\section{Debate}

Akerlof e Kranton (2000), Sen (2002b) e Davis (2011) afirmam que a identidade é um tema novo na Economia, por vezes ainda negligenciado, mesmo com a ênfase dada ao indivíduo nessa ciência. Embora essa afirmação possa ser contestada, já que escritos da economia clássica já continham elementos associados à identidade, é possível compreender tal afirmação como o negligenciamento da identidade pelas abordagens contemporâneas da ciência econômica. Os autores concordam que, com a (re)introdução desse conceito, questões ligadas ao indivíduo e ao comportamento humano serão melhores compreendidas. Contudo, a ambiguidade do conceito de identidade, oriunda das diferentes vertentes filosóficas, sociológicas, psicológicas e econômicas, faz com que as abordagens de Akerlof e Kranton, Sen e Davis possuam pontos de divergência, conforme o Quadro 1. 
Quadro 1 - Abordagens contemporâneas da identidade na Economia

\begin{tabular}{|c|c|c|c|c|c|}
\hline Autor (es) & $\begin{array}{l}\text { Identi- } \\
\text { dades }\end{array}$ & $\begin{array}{c}\text { Incorpo- } \\
\text { ração na } \\
\text { abordagem }\end{array}$ & $\begin{array}{l}\text { Influência da } \\
\text { identidade } \\
\text { sobre o com- } \\
\text { portamento }\end{array}$ & $\begin{array}{l}\text { Múltiplas } \\
\text { identidades }\end{array}$ & Natureza \\
\hline $\begin{array}{c}\text { Akerlof e } \\
\text { Kranton }\end{array}$ & $\begin{array}{l}\text { Identida- } \\
\text { de social. }\end{array}$ & $\begin{array}{c}\text { Função de } \\
\text { utilidade = } \\
\text { preferências } \\
\text { + identida- } \\
\text { de (catego- } \\
\text { rias sociais). }\end{array}$ & $\begin{array}{c}\text { Ação própria; } \\
\text { ação dos ou- } \\
\text { tros; escolha } \\
\text { sobre a própria } \\
\text { identidade; } \\
\text { influências } \\
\text { da sociedade } \\
\text { nas categorias } \\
\text { sociais e prescri- } \\
\text { ções. }\end{array}$ & $\begin{array}{c}\text { Única } \\
\text { identidade } \\
\text { social em um } \\
\text { único tempo. }\end{array}$ & $\begin{array}{l}\text { Mutável } \\
\text { e limitada } \\
\text { pelo indiví- } \\
\text { duo e pelos } \\
\text { outros. }\end{array}$ \\
\hline Sen & $\begin{array}{l}\text { Iden- } \\
\text { tidade } \\
\text { pessoal e } \\
\text { social. }\end{array}$ & $\begin{array}{l}\text { Commit- } \\
\text { ment }\end{array}$ & $\begin{array}{l}\text { O indivíduo } \\
\text { é guiado pelo } \\
\text { autointeresse } \\
\text { e segue regras } \\
\text { do grupo que } \\
\text { se identifica; } \\
\text { a identidade } \\
\text { pessoal implica } \\
\text { na autonomia, } \\
\text { dada pelo julga- } \\
\text { mento do pró- } \\
\text { prio indivíduo. }\end{array}$ & $\begin{array}{c}\text { Múltiplas } \\
\text { identidades } \\
\text { sociais simul- } \\
\text { taneamente, } \\
\text { que podem } \\
\text { entrar em } \\
\text { conflito entre } \\
\text { si. }\end{array}$ & $\begin{array}{c}\text { Ambas são } \\
\text { mutáveis, } \\
\text { via escolha } \\
\text { e avaliação } \\
\text { do indiví- } \\
\text { duo; são } \\
\text { limitadas } \\
\text { pelos ou- } \\
\text { tros e pelas } \\
\text { caracterís- } \\
\text { ticas dos } \\
\text { indivíduos. }\end{array}$ \\
\hline Davis & $\begin{array}{l}\text { Iden- } \\
\text { tidade } \\
\text { pessoal, } \\
\text { indivi- } \\
\text { dual e } \\
\text { social. }\end{array}$ & $\begin{array}{l}\text { Abordagem } \\
\text { filosófica dos } \\
\text { critérios de } \\
\text { identidade } \\
\text { (individua- } \\
\text { lização e } \\
\text { reidentifica- } \\
\text { ção) }\end{array}$ & $\begin{array}{l}\text { O indivíduo é } \\
\text { socialmente in- } \\
\text { corporado, mas } \\
\text { mantém aspec- } \\
\text { tos de singulari- } \\
\text { dade (pessoal e } \\
\text { individual). }\end{array}$ & $\begin{array}{c}\text { Múltiplas } \\
\text { identidades } \\
\text { sociais simul- } \\
\text { taneamente, } \\
\text { que podem } \\
\text { entrar em } \\
\text { conflito com } \\
\text { a identidade } \\
\text { pessoal e } \\
\text { individual. }\end{array}$ & $\begin{array}{l}\text { Mutáveis, } \\
\text { com as- } \\
\text { pectos } \\
\text { singulares } \\
\text { (pessoal e } \\
\text { individual) } \\
\text { e deter- } \\
\text { minados } \\
\text { socialmen- } \\
\text { te. }\end{array}$ \\
\hline
\end{tabular}

Fonte: Elaboração própria.

As três abordagens estão preocupadas com a identidade social do indivíduo. Sen (1998, 2002b) e Davis (2009) entendem que os indivíduos se identificam com múltiplas identidades relacionadas com questões culturais, profissionais, de gênero, etc. Akerlof e Kranton (2000) afirmam que os indivíduos podem possuir várias categorias sociais, baseadas nas identidades sociais, porém seu modelo considera uma única identidade social em um único tempo. Essa identidade única dependerá da situação em que o indivíduo está inserido e é mutável. Sen (1998, 2002a, 2008b) e Davis (2011) enfatizam a multiplicidade de identidades sociais, 
a importância da escolha das identidades e são a favor dos demais conceitos de identidades: identidades pessoais para Sen (1998) e pessoais e individuais para Davis (2011). A abordagem da identidade de Akerlof e Kranton, por ser oriunda da psicologia, enfatiza a identidade social e ignora a identidade pessoal (ou apenas a considera incluída na função de utilidade do indivíduo) (DAVIS, 2006, 2011) - a base psicológica utilizada por Akerlof e Kranton é analisada a seguir.

Akerlof e Kranton (2000), Sen (2002b) e Davis (2003; 2011) introduzem o conceito de identidade na Economia por meios diferentes. Sen (2002b) incorpora a identidade como uma influência no comportamento humano e na realização dos objetivos do indivíduo, mas não a insere em nenhum modelo econômico ou função de utilidade, como realizado por Akerlof e Kranton (2000). Davis (2003, 2011) possui uma abordagem filosófico-ontológica, na qual investiga o indivíduo na Economia, via dois critérios de identidade: individualização, que explica como os indivíduos podem ser distintos uns dos outros, ou seja, como distinguir os indivíduos como independentes uns dos outros; e a reidentificação, que capta se o indivíduo é um ser distinto, separado dos outros.

Quanto às influências da identidade sobre o comportamento humano, Davis (2009) mostra que o comportamento dos indivíduos é influenciado pelo meio em que vive, sendo um indivíduo socialmente incorporado, mas que consegue manter a identidade pessoal e individual dentro da sociedade. Por outro lado, a abordagem de Akerlof e Kranton (2000) propõe uma função de utilidade composta por preferências e identidades, esta que é a central para o comportamento do indivíduo, por ser sua principal motivação. A identidade pode influenciar o comportamento do indivíduo por quatro maneiras distintas: a ação do próprio indivíduo, a ação dos outros, via escolha e influenciado pela sociedade. Na abordagem de Sen (2002b), o indivíduo não é guiado exclusivamente pelo autointeresse, mas também é influenciado pela ação de grupos e indivíduos que se identifica. Também é importante, para Sen (2002b), a identidade pessoal, relacionada com a autonomia do indivíduo, no sentido de ser capaz de tomar decisões sobre si mesmo e escolher em quais grupos sociais deseja estar inserido.

Um ponto importante para entender a conceituação de identidade de Akerlof e Kranton diz respeito à base psicológica sobre a qual a abordagem repousa. Akerlof e Kranton (2000, 2005) se remetem explicitamente à teoria da dissonância cognitiva. Embora os estudos de Leon Festinger (1919-1989) não sejam citados pelos autores, Festinger é o pai fundador da teoria da dissonância cognitiva. ${ }^{15}$ Festinger (1957) apresenta o exemplo clássico de dissonância cognitiva, o exemplo do fumante. De acordo com Festinger (1957), um fumante que descobriu que

15 Festinger é um pesquisador de extrema importância no campo da psicologia e sua teoria da dissonância cognitiva é reconhecida como essência para a ciência. A importância de Festinger pode ser percebida em uma publicação denominada "The 100 most eminent psychologists of the 20th century", na qual Festinger ocupa a quinta posição. Ver Haggbloom et al. (2002). 
fumar faz mal para a saúde está em uma situação de dissonância cognitiva. Em dissonância cognitiva, os tomadores de decisão sentem uma pressão para criar novas relações consonantes, pois o comportamento - fumar - não é mais compreendido como algo desejável ou aceitável. O fumante deve estabelecer uma nova consonância cognitiva. Festinger (1957) destaca que existem duas possibilidades para que o fumante estabeleça uma nova consonância cognitiva: a) uma mudança de comportamento devido à compreensão dos malefícios do ato de fumar, consequentemente o fumante pararia de fumar (ou tentaria parar); b) uma mudança de comportamento que reforce o ato de fumar, nesse caso a consonância seria estabelecida com os bons efeitos do ato de fumar. Nos dois cenários, a nova consonância cognitiva não é estabelecida automaticamente.

É fundamental destacar que Festinger (1957) afirma que cultura e grupos podem influenciar no estabelecimento de consonância cognitiva, mas não aplica essa influência em sua teoria. Na maior parte das sociedades contemporâneas, tomadores de decisão já possuem informações sobre os malefícios do ato de fumar antes de se tornarem fumantes. Dessa forma, há a exclusão do processo de socialização prévio e sempre há o processo de escolha própria do tomador de decisão. Há elementos presentes no cerne da abordagem de Akerlof e Kranton, que, conforme destacado por Fine (2009), possibilitam que Akerlof e Kranton incorporem a identidade no mainstream sem perturbações no domínio da disciplina. De outra forma, segundo Possas (1997), Akerlof e Kranton trazem a identidade para dentro da "cheia do mainstream". ${ }^{16}$ A cheia do mainstream gerada por Akerlof e Kranton pode ser verificada na forma pela qual a dissonância cognitiva foi incorporada no modelo. Para Akerlof e Kranton (2000), a tensão gerada pela dissonância cognitiva é interpretada como perda de utilidade, de modo que a maximização da utilidade é dada pela minimização dessas tensões.

O tratamento dado aos elementos psicológicos nos quais a identidade se baseia é mais complexo tanto em Sen quanto em Davis. Ambos destacam que existe a cognição do tomador de decisão, que diz respeito a características e trajetórias pessoais do indivíduo, mas que também há uma cognição social, que diz respeito ao ambiente de tomada de decisão. Davis é mais explícito na consideração de conjuntos cognitivos distintos ao destacar que a identidade pessoal (eu) e a identida-

16 A "cheia do mainstream" é uma metáfora apresentada por Possas (1997). Nas palavras de Possas (1997, p. 13): "[s]eguindo a imagem fluvial, são dois os efeitos típicos de uma cheia: o aumento da correnteza e a inundação das margens. O 'mainstream' da ciência econômica tem mostrado fortemente ambos os efeitos na última década e meia, aproximadamente. De um lado, a corrente tornou-se mais caudalosa - arrastando uma proporção crescente e inusitada de economistas profissionais e acadêmicos - e mais rápida - abrindo sua agenda, fechando questões pendentes e uniformizando o discurso, cada vez mais formalizado, num ritmo sem precedentes, indicativo de grande vitalidade. De outro, vários temas relevantes considerados marginais ou intratáveis, e por isso relegados à heterodoxia (ou ao limbo), passaram a ser incorporados, ganhando o status de objetos cientificamente sérios." 
de individual (ele) formam a identidade social (nós). Características pessoais e do ambiente de tomada de decisão são levadas em consideração no estabelecimento da habilidade cognitiva do tomador de decisão. Sen também o faz ao destacar a possibilidade de múltiplas identidades sociais através das quais o autointeresse do tomador de decisão o guia.

No entanto, embora tanto a abordagem de Sen quanto a de Davis geram um arcabouço analítico mais complexo que a apresentada por Akerlof e Kranton, tal complexidade gera dificuldades analíticas. Ao se delinear mais de uma identidade baseada em diferentes cognições, a capacidade de se analisar aquilo que motivou uma determinada decisão se reduz devido à existência de uma ampla sorte de combinação de fatores. Consequentemente, dentre os estudos econômicos contemporâneos que analisam a identidade, tem-se: a) a abordagem do mainstream, que possui uma grande simplificação analítica e uma grande possibilidade de aplicações empíricas; e b) abordagens não mainstream, baseadas em análises mais complexas e aplicações empíricas também distantes da trivialidade.

\section{Considerações Finais}

As três abordagens têm por conclusão que, para se compreender melhor os fenômenos econômicos que são resultados do comportamento humano, a identidade é central para a análise econômica. Ao se considerar a identidade, a análise econômica tende a sofrer mudanças, que podem fornecer respostas mais precisas aos problemas que incluem o elemento "indivíduo", pois a identidade auxilia a fornecer o significado do que é ser um indivíduo. Porém, considerar a identidade também aumenta a complexidade da abordagem da tomada da decisão.

O modelo mainstream de Akerlof e Kranton torna a identidade central para o comportamento humano e para a escolha do indivíduo. Na sua visão, a incorporação da identidade na Economia aborda como o comportamento dos indivíduos dentro de grupos sociais influenciam os comportamentos e as escolhas individuais, ou seja, como as interações entre indivíduos limitam ou maximizam a sua utilidade.

$\mathrm{Na}$ abordagem de Sen de identidade, o commitment é uma nova forma de se pensar em comportamento humano, que se relaciona com a identidade social e pessoal. Os indivíduos são influenciados pela sociedade à qual estão inseridos e são capazes de formar comprometimentos com os outros indivíduos. Para o autor, o indivíduo possui múltiplas identidades relacionadas com os diferentes grupos sociais aos quais se identifica e às diversas situações, além de ter autonomia para escolher quem deseja ser, ou seja, escolher a sua própria identidade (pessoal e social). Com a introdução da identidade, a principal contribuição de Sen é expandir a análise de bem-estar dos indivíduos ao considerar as influências dos outros indivíduos sobre o seu comportamento. 
A abordagem de Davis trata as identidades em três diferentes níveis: a identidade pessoal, a individual e a social, sendo esta construída através das duas primeiras. As identidades individuais permitem a individualização do indivíduo em contextos sociais, enquanto que as identidades pessoais são interpretadas pelas características de cada indivíduo e fazem parte das identidades individuais. Para o autor, a incorporação da identidade na Economia enriqueceria o conceito de indivíduo por abranger tanto os aspectos individuais quanto os sociais, dado que o indivíduo não é um ser isolado, mas que interage em ambiente social.

\section{Referências}

AKERLOF, G. A. The market for "lemons": quality uncertainty and the market mechanism. The Quarterly Journal of Economics, v. 84, n. 3, p. 488-500, 1970

AKERLOF, G. A. Behavioral macroeconomics and macroeconomic behavior. The American Economic Review, v. 92, n. 3, p. 411-433, 2002.

AKERLOF, G. A.; KRANTON, R. E. Economics and Identity. The Quarterly Journal of Economics,v. 115, n. 3, p. 715-753, 2000.

AKERLOF, G. A.; KRANTON, R. E. Identity and schooling: some lessons for the economics of education. Journal of Economic Literature, v. 40, n. 4, p. 1167-1201, 2002.

AKERLOF, G. A.; KRANTON, R. E. Identity and the economics of organizations. The Journal of Economic Perspectives, v. 19, n. 1, p. 9-32, 2005.

AKERLOF, G. A.; KRANTON, R. E. Identity, supervision, and work groups. American Economic Review, v. 98, n. 2, p. 212-217, 2008.

COLANDER, D. The death of neoclassical economics. Journal of the History of Economic Thought, v. 22, n. 2, p. 127-143, 2000

DAVIS, J. B. Atomism, identity criteria, and impossibility logic. Methodus: Bulletin of the International Network for Economic Method, v. 4, n. 1, p. 83-87, 1992.

DAVIS, J. B. The theory of the individual in economics: identity and value. London: Routledge, 2003.

DAVIS, J. B. Identity and commitment: Sen's conception of the individual. Tinbergen Institute Discussion Paper, v. 55, n. 2, 2004.

DAVIS, J. B. The individual in economics. Amsterdam: Vossiuspers UvA, 2005. p. 1-26.

DAVIS, J. B. Social identity strategies in recent economics. Journal of Economic Methodology, v. 13, n. 3, p. 371-390, 2006.

DAVIS, J. B. Identity and individual economic agents: a narrative approach. Review of Social Economy, v. 67, n. 1, p. 71-94, 2009. 
DAVIS, J. B. The Homo economicus conception of the individual: an ontological approach. Social Science Research Network, p. 1-45, 2010.

DAVIS, J. B. Individuals and identity in economics. New York: Cambridge University Press, 2011.

DEQUECH, D. Neoclassical, mainstream, orthodox, and heterodox economics. Journal of Post Keynesian Economics, v. 30, n. 2, p. 279-302, 2007.

DEQUECH, D. Post Keynesianism, Heterodoxy and Mainstream Economics. Review of Political Economy, v. 24, n. 2, p. 353-368, 2012.

FESTINGER, L. A theory of cognitive dissonance. Stanford: Stanford University Press, 1957.

FINE, B. The economics of identity and the identity of economics? Cambridge Journal of Economics, v. 33, n. 2, p. 175-191, 2009.

HAGGBLOOM, S. J.; WARNICK, R.; WARNICK, J. E.; JONES,V. K.; YARBROUGH, G. L.;RUSSEL, T. M.;BORECKY, C. M.; MCGAHHEY, R.; POWEL, J. L.; BEAVERS, J.; MONTE, E. The 100 most eminent psychologists of the $20^{\text {th }}$ century. Review of General Psychology, v. 6, n. 2, p. 139-152, 2002.

HAUSMAN, D. S.; MCPHERSON, M. Economic nalysis, moral philosophy and public policy. 2nd ed. New York: Cambridge University Press, 2006.

KIRMAN, A. Complex economics: individual and collective rationality. New York: Routledge, 2011.

KIRMAN, A.; TESCHL, M. On the emergence of economic identity. Revue de Philosophie Économique, v. 9, p. 59-86, 2004.

MARCH, J. G. A primer on decision making: how decisions happen. New York: The Free Press, 1994.

PAREKH, B. Clearing the fog. Politics, Philosophy E Economics, v. 8, n. 3, p. 289-290, 2009a.

PAREKH, B. Logic of identity. Politics, Philosophy 8 Economics, v. 8, n. 3, p. 267-284, 2009b.

POSSAS, M. L. A cheia do "mainstream": comentário sobre os rumos da ciência econômica. Revista de Economia Contemporânea, v. 1, n. 1, p. 13-37, 1997

QIZILBASH, M. Identity, reason and choice. Economics and Philosophy, v. 30, n. 1, p. 11-33, 2014.

SEN, A. K. Rational fools: a critique of the behavior foundations of economic theory. Philosophy and Public Affairs, v. 6, n. 4, p 317-344, 1977.

SEN, A. K. Well-being, agency and freedom: the Dewey lectures. The Journal of Philosophy, v. 82, n. 4, p. 169-221, 1985.

SEN, A. K. Economic methodology: heterogeneity and relevance. Social Research, v. 56, n. $2,1989$. 
SEN, A. K. Reason before identity. New York: Oxford University Press, 1998.

SEN, A. K. Identity, identity. 2002a. p. 150-154. Disponível em: https://journals.sagepub.com/ doi/pdf/10.1080/03064220208537059 . Acesso em: 09 ago. 2019.

SEN, A. K. Rationality and freedom. Cambridge: The Belknap Press of Harvard University Press, 2002b.

SEN, A. K. Why exactly is commitment important for rationality? Economics and Philosophy, v. 21 , n. 1, p. 5-14, 2005.

SEN, A. K. Identity and violence: the illusion of destiny. New York: W. W. Norton $\mathcal{E}$ Company, 2007.

SEN, A. K. Sobre ética e economia. São Paulo: Companhia da Letras, 2008a.

SEN, A. K. Violence, identity and poverty. Journal of Peace Research, v. 45, n. 1, p. 5-15, 2008b.

SEN, A. K. The fog of identity. Politics, Philosophy E Economics, v. 8, n. 3, p. 285-288, 2009.

SEN, A. K. Justice and identity. Economics and Philosophy, v. 30, n. 1, p. 1-10, 2014.

SIMON, H. Bounded rationality in social science: today and tomorrow. Mind 8 Society, v. 1, n. 1, p. 25-39, 2000.

SIMON, H. Organizations and markets. Journal of Economic Perspectives, v. 5, n.2, p. 25-44, 1991.

SPENCE, A. Job market signaling. Quarterly Journal of Economics, v. 87, n. 3, p. 355-374, 1973.

STIGLITZ, J.; ROTHSCHILD, M. Equilibrium in competitive insurance markets: an essay on the economics of imperfect information. Quarterly Journal of Economics, v. 90, n. 4, p. 629649, 1976.

THERÉT, B. As instituições entre as estruturas e as ações. Revista Lua Nova, n. 58, p. 224-254, 2003.

Recebido em: 16/08/2017.

Aceito em: 01/05/2018. 